\title{
Relações entre crescimento vegetativo e acúmulo de nitrogênio em duas cultivares de arroz com arquiteturas contrastantes
}

\author{
Marcel Giovanni Costa França ${ }^{1,3}$, Adelson Paulo Araújo², Roberto Oscar Pereyra Rossiello² e \\ Flávia Toledo Ramos ${ }^{2}$
}

Recebido em 3/01/2006. Aceito em 23/04/2007

\begin{abstract}
RESUMO - (Relações entre crescimento vegetativo e acúmulo de nitrogênio em duas cultivares de arroz com arquiteturas contrastantes). Foram estudados, mediante análise do crescimento, o acúmulo de biomassa e nitrogênio $(\mathrm{N})$ e sua eficiência de utilização, durante o estádio de crescimento vegetativo, em duas cultivares de arroz (Oryza sativa L.) com arquiteturas contrastantes: IAC-4440, cultivar do grupo moderno para inundação, e Comum Branco, cultivar local rústica de sequeiro. Em experimento com solução nutritiva, foram efetuadas sete coletas, dos 25 aos 85 dias de crescimento, quando foram quantificados a área foliar, a massa seca e o conteúdo de $\mathrm{N}$ nas porções vegetativas. A cultivar IAC apresentou maiores valores de biomassa na fase inicial do crescimento, enquanto Comum Branco maiores valores na fase final do experimento. IAC-4440 teve maiores área foliar, área foliar específica e relação raiz: parte aérea do que Comum Branco. As cultivares tiveram o mesmo acúmulo total de N, mas Comum Branco apresentou valores superiores na taxa de assimilação líquida e na taxa de utilização específica de $\mathrm{N}$, indicando melhor utilização do $\mathrm{N}$ absorvido. As maiores taxas de crescimento de Comum Branco estiveram associadas aos seus maiores teores de $\mathrm{N}$ por unidade de área foliar, mas não aos teores de $\mathrm{N}$ por unidade de massa foliar, superiores em IAC-4440. Os resultados indicam que uma cultivar local rústica de arroz pode servir como fonte de eficiência para utilização de N.
\end{abstract}

Palavras-chave: análise de crescimento, área foliar, ecotipo, Oryza sativa

\begin{abstract}
The relationship between vegetative growth and nitrogen accumulation in two rice cultivars with contrasting architecture). Accumulation of biomass and nitrogen $(\mathrm{N})$ during the vegetative growth stage and $\mathrm{N}$ utilization efficiency were evaluated using plant growth analysis in two rice (Oryza sativa L.) cultivars with contrasting architecture: IAC-4440, a modern lowland cultivar, and Comum Branco, a rustic upland cultivar. An experiment with nutrient solution involved seven harvests that took place in the 25 to 85 growthdays period, where leaf area, dry mass, and $\mathrm{N}$ content in vegetative plant parts were measured. Cultivar IAC had higher biomass at the beginning of the growth cycle, whereas Comum Branco had higher values at the end of the experiment. IAC-4440 had greater leaf area, specific leaf area and root-to-shoot ratio than Comum Branco. The cultivars accumulated the same amount of N, but Comum Branco showed greater net assimilation rate and specific $\mathrm{N}$ utilization rate, indicating greater utilization of the $\mathrm{N}$ absorbed. Higher growth rates of Comum Branco were associated with higher $\mathrm{N}$ concentration per unit leaf area, but not with $\mathrm{N}$ concentration per unit leaf mass which were superior in IAC-4440. These results indicate that a local rustic cultivar of rice can provide a source of $\mathrm{N}$ utilization efficiency.
\end{abstract}

Key words: ecotype, growth analysis, leaf area, Oryza sativa

\section{Introdução}

As relações entre o acúmulo de biomassa e de nitrogênio $(\mathrm{N})$ em cultivos dependem de vários processos fisiológicos, dentre os quais a absorção de $\mathrm{N}$, a assimilação de carbono (C) via fotossíntese, a taxa de crescimento da cultura, e a alocação de $\mathrm{C}$ e $\mathrm{N}$ entre órgãos da planta e entre plantas do dossel (Gastal \& Lemaire 2002). A capacidade fotossintética vegetal, e o posterior acúmulo e distribuição de massa seca e $\mathrm{N}$, estão fortemente relacionados a fatores ambientais como as condições climáticas e edáficas, e a disponibilidade de nutrientes (Ntanos \& Koutroubas 2002). Entretanto, esses fenômenos são também controlados por fatores internos da planta, como as diferenças na morfologia e na fisiologia da folha, que são características dependentes do genótipo, e também do estágio de desenvolvimento vegetal (Hasegawa \& Horie 1996).

No arroz (Oryza), com seu grande número de espécies e cultivares, são encontrados genótipos que apresentam diferentes taxas de fotossíntese por

\footnotetext{
1 Instituto de Ciências Biológicas, Departamento de Botânica, Universidade Federal de Minas Gerais, Av. Antônio Carlos, 6627, Pampulha, 31270-901 Belo Horizonte, MG, Brasil

2 Universidade Federal Rural do Rio de Janeiro, Instituto de Agronomia, Departamento de Solos, BR 465 km 7, 23890-000 Seropédica, RJ, Brasil

3 Autor para correspondência: marcel@icb.ufmg.br
} 
unidade de área foliar, sendo essas taxas relativamente mais altas entre as espécies de $O$. sativa cultivadas (Cook \& Evans 1983). Murata \& Matsushima (1975) destacaram que as taxas fotossintéticas nos estágios iniciais do crescimento apresentam uma correlação positiva com o conteúdo de $\mathrm{N}$ por unidade de área foliar, indicando que um aumento na espessura foliar, associado a um aumento do conteúdo de $\mathrm{N}$ foliar, propiciaria aumento nas taxas fotossintéticas. Sinclair \& Horie (1989), apresentando resultados de vários autores, mostraram que a taxa máxima de fotossíntese em arroz, sob condições de saturação de luz, aumentava linearmente com o teor de $\mathrm{N}$ foliar, até um valor de $1,6 \mathrm{~g} \mathrm{~N} \mathrm{~m}^{-2}$ de folha, e depois declinava de forma curvilínea. $\mathrm{O}$ aumento excessivo do teor de $\mathrm{N}$ foliar favorece principalmente o aumento da área foliar (Sarkar et al. 2002), que por efeitos de auto-sombreamento e aumento da resistência estomática, conduzem a declínios da fotossíntese líquida do dossel (Murata 1969). Estas condições podem permitir a ação dos fatores que favorecem ao tombamento da planta ao final do ciclo, devido ao tamanho e peso da panícula e, também pelo porte e arquitetura da planta.

Norman et al. (1992) destacaram que o acúmulo de $\mathrm{N}$ nas folhas, e a posterior distribuição para as porções reprodutivas, representam processos determinantes na produção de grãos em arroz, pois as folhas são a principal estrutura de estoque de $\mathrm{N}$ (Mae 1997). Adicionalmente, na metade do período de perfilhamento e 10 dias antes da formação da panícula, período de máxima absorção de $\mathrm{N}, 65 \%$ do $\mathrm{N}$ total da parte aérea, localiza-se em lâminas foliares (Ntanos \& Koutroubas 2002). Desta forma, algumas características buscadas em programas de melhoramento, visando o aumento da produtividade na cultura do arroz, tais como o ângulo de inserção e a densidade de folhas, um porte baixo, um menor número de perfilhos (Matsushima 1979), e a espessura foliar (Villar et al. 2005), podem ser modificadas pela disponibilidade de $\mathrm{N}$ e pela intensidade de luz, que afetam a quantidade de folhas, a arquitetura da planta e outras características morfológicas (Akita 1995).

Cultivares locais de arroz, como as tradicionalmente plantadas por pequenos produtores do Estado do Maranhão, podem apresentar elevada eficiência de utilização do $\mathrm{N}$ absorvido para produção de biomassa e de grãos, por sua maior adaptação a solos de baixa fertilidade (Ferraz Junior et al. 1997). O objetivo deste trabalho foi avaliar, por meio da análise quantitativa do crescimento, as diferenças no acúmulo e distribuição de massa seca e $\mathrm{N}$ em duas cultivares de arroz com características morfológicas contrastantes, e possivelmente com diferentes eficiências de utilização de $\mathrm{N}$ para produção de biomassa, cultivadas em solução nutritiva com ótima disponibilidade nutricional, durante a fase vegetativa do crescimento.

\section{Material e métodos}

Foram estudadas duas cultivares de arroz (Oryza sativa L.) com similar duração do ciclo de crescimento, mas com distintas características ecofisiológicas. IAC-4440, oriunda do Instituto Agronômico de Campinas, é uma cultivar do grupo moderno, que responde à adubação nitrogenada e apresenta características que favorecem o crescimento em condições alagadas, com porte baixo e folhas estreitas e eretas, apresentando elevado perfilhamento e alto potencial produtivo, melhor evidenciado com altos níveis de nutrientes no solo (Usberti Filho et al. 1986), e a cultivar Comum Branco, considerada uma "variedade crioula", é um genótipo rústico de sequeiro, oriunda do Estado do Maranhão (Coradin \& Fonseca 1982), de porte alto, baixo perfilhamento, folhas largas e decumbentes, possuindo uma arquitetura inadequada à maximização da produtividade, devido ao porte alto que favorece ao acamamento e ao seu hábito decumbente que restringe a captação de radiação solar (Akita 1995). Entretanto, Comum Branco destaca-se pela elevada tolerância ao alumínio e alto teor de proteína no grão (Santos M.L.M. 1991; Ferraz Júnior A.S.L. 1993, dissertações de mestrado UFRRJ, dados não publicados).

Sementes desinfestadas superficialmente foram germinadas em areia lavada e autoclavada. Aos 10 dias após plantio (DAP), foram transferidas duas plântulas por vaso plástico, contendo 3,3 L da solução nutritiva descrita por Furlani et al. (1986), a meia força iônica. Aos 15 DAP, a solução foi trocada por uma de força iônica total, ajustada a pH 5,0 $\pm 0,2$, que continha $60 \mathrm{mg} \mathrm{N} \mathrm{L}{ }^{-1}$, com uma relação $\mathrm{N}_{-} \mathrm{NO}_{3}^{-}: \mathrm{N}^{-} \mathrm{NH}_{4}{ }^{+}$de 4. Aos $45 \mathrm{DAP}$ as plantas foram transferidas para vasos contendo $12 \mathrm{~L}$ de solução nutritiva. $\mathrm{O}$ volume de água deionizada necessário para compensar perdas por transpiração foi adicionado diariamente. $\mathrm{O}$ experimento foi conduzido em casa de vegetação, onde a temperatura média do ar oscilou entre 31 e $35^{\circ} \mathrm{C}$, e a umidade relativa do ar entre 37 e $96 \%$. O fluxo de fótons, medido às 12 horas, teve valor de $1350 \pm$ $640 \mu \mathrm{mol}$ fótons $\mathrm{m}^{-2} \mathrm{~s}^{-1}$. 
Foram realizadas sete coletas em intervalos de dez dias, entre os 25 e 85 DAP (aproximadamente duas semanas antes da floração), com quatro vasos coletados por cultivar. O estudo limitou-se até aos 85 dias após plantio, porque até esta fase dá-se o acúmulo de mais de $85 \%$ do total de $\mathrm{N}$ absorvido por IAC-4440 e Comum Branco durante um ciclo de 135 dias (França et al. 1999). Por ocasião de cada coleta, foi trocada a solução nutritiva dos vasos restantes. Em cada coleta, as plantas foram separadas em raízes, lâminas foliares e colmos + bainhas. Até a $2^{\mathrm{a}}$ coleta, a área foliar foi determinada em todas as folhas utilizando-se um medidor fotoelétrico de área (LI 3000, LI-COR). A partir da 3 a coleta, a área foliar foi determinada retirando-se de cada planta 10 discos foliares com área superficial conhecida, que foram secos e pesados; a partir da área foliar dos discos e sua massa seca, juntamente com a massa seca total das folhas da planta, determinou-se a área foliar total. A área foliar específica foi obtida pela divisão da área foliar pela massa seca foliar. $\mathrm{O}$ material de cada porção vegetal foi secado em estufa, pesado e moído, sendo determinado o teor de $\mathrm{N}$ pelo método semi-micro Kjeldhal. O conteúdo de $\mathrm{N}$ de cada porção foi obtido pelo produto entre o teor e a massa seca.

As variâncias dos dados primários mostraram heterogeneidade entre as coletas, exceto para os teores de $\mathrm{N}$ nas folhas; desta forma, os dados heterocedásticos foram transformados em logaritmo natural previamente à análise estatística (Araújo 2003). A análise de variância foi efetuada como um fatorial $2 \times$ 7 (cultivar $\times$ época de coleta), com quatro repetições, em um delineamento completamente casualisado; foram também efetuados contrastes entre cultivares dentro de cada coleta através da decomposição das somas dos quadrados (teste F com um grau de liberdade). Foram ajustadas funções matemáticas aos dados primários de área foliar, massa seca e conteúdo de N, por processo iterativo (programa SAEG, Fundação Artur Bernardes, UFV), escolhendo-se o modelo de Gompertz. A partir das funções ajustadas para os dados primários foram obtidas, por derivação, as taxas de crescimento relativo (TCR), a taxa de assimilação líquida (TAL), e a taxa de utilização específica de N (TUE), segundo as expressões (Hunt 1982): $\mathrm{TCR}=1 / \mathrm{M} \cdot \mathrm{dM} / \mathrm{dT}\left(\mathrm{em} \mathrm{g} \mathrm{g}^{-1} \mathrm{dia}^{-1}\right)$, $\mathrm{TAL}=1 / \mathrm{A} \cdot \mathrm{dM} / \mathrm{dT}\left(\mathrm{em} \mathrm{g} \mathrm{m}{ }^{-2} \mathrm{dia}^{-1}\right), \mathrm{e} \mathrm{TUE}=1 / \mathrm{N}$. $\mathrm{dM} / \mathrm{dT}\left(\mathrm{em} \mathrm{g} \mathrm{g}^{-1} \mathrm{~N} \mathrm{dia}{ }^{-1}\right)$, onde $\mathrm{M}$ é a massa seca total, $\mathrm{A}$ a área foliar, $\mathrm{N}$ o total de $\mathrm{N}$ acumulado na planta, e T o tempo (em DAP).

\section{Resultados e discussão}

Acumulação e partição da massa seca - A análise de variância identificou interações significativas entre cultivares e época de coleta para o acúmulo de massa seca de caule, folha, raiz e total, demonstrando diferentes ritmos de acúmulo de biomassa entre as cultivares, nas diferentes porções vegetativas. As duas cultivares tiveram acúmulo de massa de folha similar até os 45 DAP, mas a partir daí Comum Branco foi superior a IAC-4440 (Tab. 1). O acúmulo de massa seca em colmos foi superior na cultivar IAC-4440 na fase inicial do crescimento, mas a partir dos 65 DAP Comum Branco manteve médias superiores (Tab. 1), pois neste período, que corresponde ao chamado "emborrachamento" na cultura do arroz (Fornasieri Filho \& Fornasieri 1993), o alongamento dos colmos em Comum Branco foi bastante superior. No início do período amostral, IAC-4440 teve valores superiores de massa seca de raízes (Tab. 1). IAC-4440 obteve maiores acúmulos de biomassa total nos estádios iniciais do crescimento, mas Comum Branco foi superior a partir dos 75 DAP. Em função dos ritmos diferenciados de acúmulo de massa seca nas diferentes porções vegetativas, a cultivar Comum Branco apresentou menor razão raiz: parte aérea (Tab. 1).

As duas cultivares tiveram elevado perfilhamento, sendo que IAC-4440 teve em média $120 \%$ mais perfilhos que Comum Branco (Tab. 1). Este excessivo perfilhamento reflete especificamente uma condição de casa de vegetação em condições nutricionais ótimas. Cabe ressaltar que grande parte destes perfilhos se tornará inviáveis ou inférteis, constituindo um grande dreno metabólico de massa seca, já que admite-se como ideal de 2 a 6 perfilhos para a planta de arroz, que corresponderiam a uma maior quantidade de perfilhos viáveis e maior número de plantas em condições de campo.

Considerando que a interação significativa entre época de coleta e cultivar, na análise de variância dos dados de biomassa total transformados em logaritmo natural, demonstra diferenças significativas entre cultivares na taxa de crescimento relativo (Araújo 2003), pode-se afirmar que a cultivar Comum Branco apresentou menores valores da taxa de crescimento relativo até aos 39 DAP, quando então seus valores tornaram-se superiores a IAC-4440 até o final do experimento (Fig. 1). Isto reflete a menor produção de biomassa total de Comum Branco no início do experimento, e sua maior biomassa nos estádios posteriores (Tab. 1). 
Tabela 1. Massa seca de colmo, folha, raiz e total, número de perfilhos, área foliar, área foliar específica e razão raiz: parte aérea das cultivares de arroz Comum Branco (CB) e IAC-4440 (IAC), em sete épocas de coleta (médias de quatro repetições dos dados originais, que foram transformados em logaritmo natural antes da análise de variância).

\begin{tabular}{|c|c|c|c|c|c|c|c|c|}
\hline \multirow[t]{2}{*}{ Dias após plantio } & \multicolumn{2}{|c|}{$\begin{array}{l}\text { Massa de colmo } \\
\quad\left(\mathrm{g} \mathrm{planta}^{-1}\right)\end{array}$} & \multicolumn{2}{|c|}{$\begin{array}{l}\text { Massa de folha } \\
\quad\left(\mathrm{g} \mathrm{planta}^{-1}\right)\end{array}$} & \multicolumn{2}{|c|}{$\begin{array}{l}\text { Massa de raiz } \\
\left(\mathrm{g} \mathrm{planta}^{-1}\right)\end{array}$} & \multicolumn{2}{|c|}{$\begin{array}{c}\text { Massa total } \\
\left(\mathrm{g} \mathrm{planta}^{-1}\right)\end{array}$} \\
\hline & $\mathrm{CB}$ & IAC & $\mathrm{CB}$ & IAC & $\mathrm{CB}$ & IAC & $\mathrm{CB}$ & IAC \\
\hline 25 & 0,1 & 0,1 & 0,1 & 0,1 & 0,05 & 0,04 & 0,3 & 0,3 \\
\hline 35 & 0,6 & $0,8^{*}$ & 0,8 & 0,9 & 0,30 & $0,50 *$ & 1,7 & $2,2^{*}$ \\
\hline 45 & 3,3 & $4,0^{*}$ & 2,8 & 2,7 & 1,70 & $2,10 *$ & 7,7 & 8,7 \\
\hline 55 & 10,2 & 9,8 & 6,7 & $5,5^{*}$ & 5,60 & 5,70 & 22,4 & 21,1 \\
\hline 65 & 21,6 & 18,9 & 14,0 & $11,0^{*}$ & 10,00 & 10,10 & 45,6 & 40,1 \\
\hline 75 & 34,5 & $28,0 *$ & 21,7 & $17,3^{*}$ & 17,40 & 16,10 & 73,6 & $61,5^{*}$ \\
\hline 85 & 58,5 & 50,0 & 28,8 & $24,3^{*}$ & 20,30 & 19,20 & 107,8 & 93,5 \\
\hline \multirow[t]{2}{*}{ Dias após plantio } & \multicolumn{2}{|c|}{$\begin{array}{l}\text { Número de perfilhos } \\
\qquad\left(\mathrm{g} \mathrm{planta}^{-1}\right)\end{array}$} & \multicolumn{2}{|c|}{$\begin{array}{l}\text { Área foliar } \\
\left(\mathrm{m}^{2} \text { planta }^{-1}\right)\end{array}$} & \multicolumn{2}{|c|}{$\begin{array}{l}\text { Área foliar específica } \\
\left(\mathrm{cm}^{2} \mathrm{~g}^{-1}\right)\end{array}$} & \multicolumn{2}{|c|}{$\begin{array}{l}\text { Razão raiz: parte aérea } \\
\qquad\left(\mathrm{g} \mathrm{g} \mathrm{g}^{-1}\right)\end{array}$} \\
\hline & $\mathrm{CB}$ & IAC & $\mathrm{CB}$ & IAC & $\mathrm{CB}$ & IAC & $\mathrm{CB}$ & IAC \\
\hline 25 & 3 & 3 & 0,005 & 0,005 & 388 & $414^{*}$ & 0,21 & 0,20 \\
\hline 35 & 5 & $10 *$ & 0,022 & $0,026^{*}$ & 282 & $300 *$ & 0,23 & $0,27 *$ \\
\hline 45 & 11 & $16^{*}$ & 0,076 & 0,075 & 236 & $278 *$ & 0,27 & $0,31 *$ \\
\hline 55 & 17 & $32 *$ & 0,128 & 0,139 & 192 & $251^{*}$ & 0,33 & $0,37 *$ \\
\hline 65 & 26 & $50 *$ & 0,219 & 0,224 & 156 & $204 *$ & 0,28 & $0,34 *$ \\
\hline 75 & 21 & $58 *$ & 0,335 & $0,400 *$ & 154 & $230 *$ & 0,31 & $0,35 *$ \\
\hline 85 & 21 & $55^{*}$ & 0,417 & 0,452 & 145 & $186^{*}$ & 0,23 & 0,26 \\
\hline
\end{tabular}

* Diferença significativa entre cultivares pelo teste $\mathrm{F}$ a $5 \%$ de probabilidade.

A cultivar IAC-4440 teve área foliar superior a Comum Branco a partir dos 35 DAP, apesar da diferença entre cultivares ter sido significativa apenas aos 35 e 75 DAP (Tab. 1). Esta maior área foliar de IAC-4440 foi obtida principalmente a partir de uma maior área foliar específica, superior a de Comum Branco durante todo o período experimental. Sendo assim, a maior biomassa total da cultivar Comum Branco ao final do período experimental, não pode ser atribuída à sua área assimilatória, que foi menor (Tab. 1). Provavelmente, outro fator tenha contribuído com esse maior crescimento em Comum Branco, como a sua menor área foliar específica (Tab. 1), que lhe confere folhas mais espessas e, por conseguinte, uma maior quantidade de cloroplastos por unidade de área foliar, proporcionando uma maior taxa fotossintética por unidade de área foliar (Villar et al. 2005).

De fato, a taxa de assimilação líquida (TAL) da cultivar Comum Branco apresentou-se superior à de IAC-4440 a partir dos 43 DAP (Fig. 1), superioridade esta que pode ser considerada estatisticamente significativa, uma vez que a TALé obtida por derivação da curva de massa seca dividida pela área foliar, sendo estas variáveis significativamente diferentes entre as cultivares (Tab. 1; Araújo 2003). Vieira et al. (1990) encontraram valores similares da TAL para plantas de arroz aos 25 DAP em experimento de campo, mas seus valores são maiores a partir desta data. Apesar de IAC-4440 ter mantido valores superiores de área foliar ao longo do período experimental (Tab. 1), suas TCR e TAL foram inferiores às de Comum Branco (Fig. 1), o que pode ser atribuído à maior área foliar específica de IAC-4440 (Tab. 1), cujas folhas menos espessas implicariam em sacrifício de potencial fotossintético por unidade de superfície. A TAL fornece uma estimativa da fotossíntese líquida por unidade de tecido assimilatório, correspondendo ao balanço final entre a fotossíntese foliar, o consumo respiratório e a absorção de nutrientes minerais (Hunt 1982). Desta forma, pode-se admitir que o melhoramento na cultivar IAC-4440 se expressou na direção de maior eficiência de captação de energia por porte ereto de folha e maior número de perfilhos, mas não por aumento na fotossíntese líquida por unidade de área foliar. Comum Branco, apesar do seu menor potencial para produção de grãos, pode apresentar uma superioridade na 

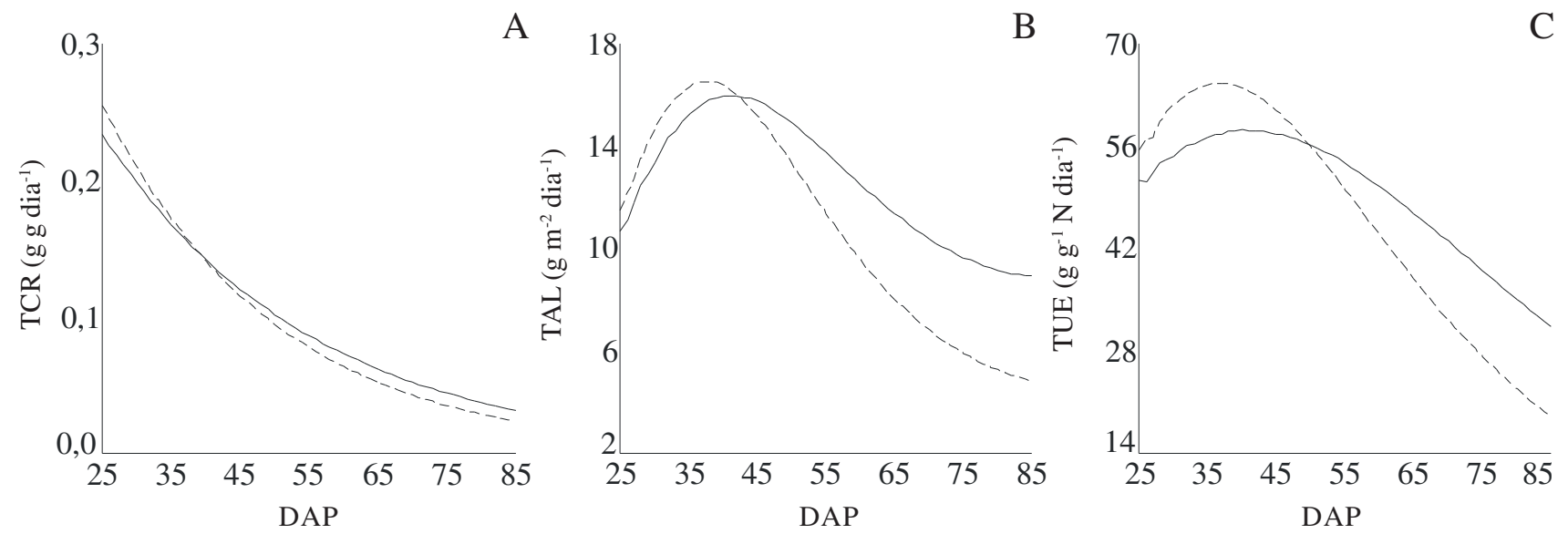

Figura 1. A. Taxa de crescimento relativo (TCR). B. Taxa de assimilação líquida (TAL). C. Taxa de utilização específica de N (TUE) das cultivares de arroz Comum Branco (-) e IAC-4440 (----) crescidas em solução nutritiva, estimadas pelo modelo de Gompertz ajustado aos dados primários de massa seca total e conteúdo total de N. DAP: Dias após plantio.

fotossintética líquida, uma característica de interesse para programas de melhoramento, desde que confirmada por estimativas diretas da assimilação de carbono através das trocas gasosas.

Acumulação e utilização de nitrogênio - Não houve diferença significativa entre cultivares para o conteúdo de $\mathrm{N}$ nas folhas e para o conteúdo total (Tab. 2), evidenciando que a arquitetura da planta não influenciou o acúmulo total de $\mathrm{N}$ pelas cultivares. $\mathrm{O}$ conteúdo de $\mathrm{N}$ nos colmos foi ligeiramente superior na cultivar IAC-4440 durante todo o período experimental (Tab. 2), o que em parte pode ser atribuído ao seu grande número de perfilhos (Tab. 1). Para o acúmulo de $\mathrm{N}$ nas raízes, apenas aos 25 DAP foram observadas diferenças significativas entre as cultivares. Os valores de conteúdo de $\mathrm{N}$ em cada porção da fitomassa são similares aos encontrados por Furlani et al. (1986), trabalhando com arroz com a mesma dose de $\mathrm{N}$ utilizada em solução nutritiva $\left(60 \mathrm{mg} \mathrm{L}^{-1}\right)$, e similares aos observados até os 55 DAP por Beri et al. (1989) em campo de arroz irrigado. Cabe destacar que a aparente similaridade na acumulação de $\mathrm{N}$ não implica em potencial produtivo similar nas cultivares estudadas, uma característica dependente principalmente da capacidade genotípica de partição do $\mathrm{N}$ acumulado préfloração entre órgãos vegetativos e reprodutivos (Norman et al. 1992).

As cultivares não diferiram no acúmulo de $\mathrm{N}$, mas sim no acúmulo de massa seca, com diferenças na alocação de biomassa em cada porção vegetativa (Tab. 1, 2). Desta forma, como a taxa de utilização específica representa a taxa de variação da biomassa por unidade de $\mathrm{N}$ acumulado, admite-se que esta seja significativamente diferente entre as cultivares (Fig. 1).

Tabela 2. Conteúdo de N no colmo, folha, raiz e total, e teor de N na folha, por unidade de massa ou por unidade de área, das cultivares de arroz Comum Branco (CB) e IAC-4440 (IAC), em sete épocas de coleta (médias de quatro repetições dos dados originais, sendo os dados de conteúdo de $\mathrm{N}$ transformados em logaritmo natural antes da análise de variância).

\begin{tabular}{|c|c|c|c|c|c|c|c|c|c|c|c|c|}
\hline \multirow{3}{*}{$\begin{array}{c}\text { Dias após } \\
\text { plantio }\end{array}$} & \multicolumn{8}{|c|}{ Conteúdo de $\mathrm{N}$ (mg N planta ${ }^{-1}$ ) } & \multicolumn{4}{|c|}{ Teor de $\mathrm{N}$ na folha } \\
\hline & \multicolumn{2}{|c|}{ Colmo } & \multicolumn{2}{|c|}{ Folha } & \multicolumn{2}{|c|}{ Raiz } & \multicolumn{2}{|c|}{ Total } & \multicolumn{2}{|c|}{$\left(\mathrm{mg} \mathrm{N} \mathrm{g}^{-1}\right)$} & \multicolumn{2}{|c|}{$\left(\mathrm{g} \mathrm{N} \mathrm{m}^{-2}\right)$} \\
\hline & $\mathrm{CB}$ & IAC & $\mathrm{CB}$ & IAC & $\mathrm{CB}$ & IAC & $\mathrm{CB}$ & IAC & $\mathrm{CB}$ & IAC & $\mathrm{CB}$ & $\overline{\text { IAC }}$ \\
\hline 25 & 3 & 3 & 7 & 7 & 1,1 & $0,9 *$ & 11 & 11 & 55,8 & $58,6^{*}$ & 1,41 & 1,43 \\
\hline 35 & 14 & $17 *$ & 36 & 38 & 6,6 & 7,2 & 56 & 62 & 46,1 & $43,1 *$ & 1,64 & $1,43^{*}$ \\
\hline 45 & 42 & 46 & 87 & 86 & 28,3 & 29,2 & 157 & 161 & 31,3 & 31,9 & 1,33 & $1,15^{*}$ \\
\hline 55 & 101 & 109 & 178 & 155 & 40,0 & 35,9 & 319 & 300 & 26,3 & $27,9 *$ & 1,39 & $1,12 *$ \\
\hline 65 & 201 & 226 & 352 & 302 & 43,4 & 46,4 & 597 & 574 & 27,4 & $30,5^{*}$ & 1,61 & $1,35^{*}$ \\
\hline 75 & 295 & 304 & 546 & 491 & 104,2 & 93,8 & 945 & 889 & 27,8 & $31,1 *$ & 1,63 & $1,23 *$ \\
\hline 85 & 360 & 392 & 562 & 564 & 129,0 & 127,1 & 1.051 & 1.083 & 22,7 & $25,8^{*}$ & 1,35 & 1,25 \\
\hline
\end{tabular}

\footnotetext{
* Diferença significativa entre cultivares pelo teste $\mathrm{F}$ a 5\% de probabilidade.
} 
A cultivar IAC-4440 mostrou ligeira superioridade na taxa de utilização específica até aos 49 DAP, mas a partir daí Comum Branco alcançou valores superiores a IAC-4440 (Fig. 1). Isto indica que uma cultivar rústica e que não passou por um planejado programa de melhoramento, pode utilizar mais eficientemente o $\mathrm{N}$ absorvido para produção de biomassa vegetativa. Ferraz Junior et al. (1997) verificaram que cultivares locais de arroz do Estado do Maranhão apresentaram maior eficiência de utilização de $\mathrm{N}$ para produção de proteínas no grão, em comparação a cultivares melhoradas, que por sua vez apresentaram maior eficência de absorção do $\mathrm{N}$ do solo. Além disto, França et al. (1999) observaram que Comum Branco apresentou maiores taxas de absorção de $\mathrm{N}$ por unidade de área radicular que IAC-4440 em solução nutritiva, reforçando a hipótese de que cultivares locais de arroz podem servir como fonte de eficiência para absorção e utilização de $\mathrm{N}$.

A disponibilidade nutricional ótima em que foi conduzido o experimento permitiu uma adequada expressão do potencial de crescimento das cultivares estudadas, como indicado pelo teor de $\mathrm{N}$ por unidade de área foliar, sempre igual ou inferior a $1,6 \mathrm{~g} \mathrm{~N} \mathrm{~m}^{-2}$ (Tab. 2), valor definido por Sinclair \& Horie (1989) como limite superior para obtenção de taxas máximas de fotossíntese em plantas de arroz. Os teores de N nas folhas das duas cultivares reduziram com o decorrer do experimento (Tab. 2), o que esteve associado com a redução ontogenética nas taxas de assimilação líquida e de utilização específica de N (Fig. 1). A cultivar IAC-4440 apresentou maiores teores de $\mathrm{N}$ nas folhas, por unidade de massa, do que Comum Branco durante quase todo período experimental (Tab. 2). Entretanto, considerando o teor de $\mathrm{N}$ por unidade de área foliar, Comum Branco apresentou maiores teores que IAC-4440 em quase todas as épocas de avaliação. Portanto, as maiores taxas de crescimento da cultivar Comum Branco ao final do período experimental (Fig. 1) foram melhor relacionadas aos maiores teores de $\mathrm{N}$ por unidade de área foliar desta cultivar, mas não aos teores de $\mathrm{N}$ por unidade de massa, que foram superiores em IAC-4440 (Tab. 1), ou seja, as taxas de crescimento estiveram mais estreitamente associadas à quantidade de $\mathrm{N}$ expressa por unidade de área, e não de massa foliar. Como a interceptação de luz é um processo dependente da área foliar, a relação entre a fotossíntese e o conteúdo de $\mathrm{N}$ foliar tem maior significado biológico, em termos de captura de energia, quando ambas as variáveis são expressas em termos de unidade de área foliar (Grindlay 1997). Além disto, as variações na área foliar específica podem confundir a interpretação das relações entre fotossíntese e acumulação de $\mathrm{N}$ nas folhas, quando esta última é expressa em termos de massa foliar (Grindlay 1997).

A taxa de utilização específica pode ser considerada um indicador adequado da eficiência de utilização de nutrientes em plantas, acompanhando a tendência de redução ontogenética da taxa de assimilação líquida (Fig. 1), ao contrário de outros índices que geralmente aumentam com a idade da planta em consequiência de efeitos de diluição (Araújo et al. 1996). Muitas das avaliações de eficiência de utilização de nutrientes em plantas correspondem a descrições estáticas que não levam em consideração diferenças na eficiência de absorção (França et al. 1999) ou diferenças em eficiência de transporte e redistribuição (Silveira \& Machado 1990). Desta forma, no presente trabalho sob condições ótimas de disponibilidade de $\mathrm{N}$ em solução nutritiva, as diferenças entre as cultivares puderam ser adequadamente expressas através das diferenças na taxa de utilização específica de N.

Os resultados obtidos indicam que uma cultivar local de arroz como Comum Branco, pode servir como fonte de eficiência para absorção e utilização de $\mathrm{N}$ em programas de melhoramento vegetal da cultura do arroz. Um ideótipo poderia contemplar plantas com folhas mais verticalizadas, como as de IAC-4440, e de menor área foliar específica, como as de Comum Branco, o que poderia conduzir a uma maior captação de luz e a maiores taxas de fotossíntese por unidade de área foliar e de $\mathrm{N}$ nos trópicos.

\section{Agradecimentos}

Ao programa de Pós-graduação em Ciência do Solo da UFRRJ, pela oportunidade de realização deste trabalho; ao CNPq, pelo auxílio financeiro.

\section{Referências bibliográficas}

Akita, S. 1995. Dry matter production of rice population. Pp. 648-661. In: T. Matsuo; K. Kumasawa; R. Ishii; K. Ishihara \& H. Hirata (eds.). Science of the rice plant. v. II - Physiology. Tokyo, FAPRC.

Araújo, A.P.; Rossiello, R.O.P.; Silva, E.M.R. \& Almeida, D.L. 1996. Growth analysis of tomato colonized with arbuscular mycorrhizal fungi. Revista Brasileira de Ciência do Solo 20: 233-240.

Araújo, A.P. 2003. Analysis of variance of primary data on plant growth analysis. Pesquisa Agropecuária Brasileira 38: 1-10. 
Beri, V.; Meelu, O.P. \& Khind, C.S. 1989. Biomass production, $\mathrm{N}$ accumulation, symbiotic effectiveness and mineralization of green manures in relation to yield of wetland rice. Tropical Agriculture 66: 11-16.

Cook, M.G. \& Evans, L.T. 1983. Some physiological-aspects of the domestication and improvement of rice (Oryza spp.). Field Crops Research 6: 219-238.

Coradin, L. \& Fonseca, J.R. 1982. Coleta de germoplasma de arroz no estado do Maranhão. Brasília, Embrapa CENARGEN (Documentos, 2).

Ferraz Junior, A.S.L.; Souza, S.R.; Fernandes, M.S. \& Rossiello, R.O.P. 1997. Eficiência do uso de nitrogênio para produção de grão e proteína por cultivares de arroz. Pesquisa Agropecuária Brasileira 32: 435-442.

Fornasieri Filho, D. \& Fornasieri, J.L. 1993. Manual da cultura do arroz. Jaboticabal: FUNEP - UNESP.

França, M.G.C.; Rossiello, R.O.P.; Zonta, E.; Araújo, A.P. \& Ramos, F.T. 1999. Desenvolvimento radicular e influxo de nitrogênio em duas cultivares de arroz. Pesquisa Agropecuária Brasileira 34: 1845-1853.

Furlani, A.M.C.; Bataglia, O.C. \& Azzini, L.E. 1986. Comportamento diferencial de linhagens de arroz na absorção e utilização de nitrogênio em solução nutritiva. Revista Brasileira de Ciência do Solo 10: 51-59.

Gastal, F. \& Lemaire, G. 2002. N uptake and distribution in crops: an agronomical and ecophysiological perspective. Journal of Experimental Botany 53: 789-799.

Grindlay, D.J.C. 1997. Towards an explanation of crop nitrogen demand based on the optimization of leaf nitrogen per unit leaf area. Journal of Agricultural Science 128: 377-396.

Hasegawa, T. \& Horie, T. 1996. Leaf nitrogen, plant age and crop dry matter production in rice. Field Crops Research 47: 107-116.

Hunt, R. 1982. Plant growth curves: The functional approach to plant growth analysis. London, Edward Arnold.

Mae, T. 1997. Physiological nitrogen efficiency in rice: Nitrogen utilization, photosynthesis, and yield potential. Plant and Soil 196: 201-210.

Matsushima, S. 1979. Cultivation technologies of ideal rice plants based on the shaped theory and soil fertility. Agriculture \& Horticulture 54: 963-970.
Murata, Y. 1969. Physiological responses to nitrogen in plants. Pp. 235-264. In: J.D. Eastin (ed.). Physiological aspects of crop yield. Madison, ASA and CSSA.

Murata, Y. \& Matsushima, S. 1975. Rice. Pp. 73-99. In: L.T. Evans (ed.). Crop physiology, some cases stories. Cambrige, University Press.

Norman, R.J.; Guindo, D.; Wells, B.R. \& Wilson, C. E. 1992. Seasonal accumulation and partitioning of N-15 in rice. Soil Science Society of American Journal 56: 1521-1526.

Ntanos, D.A. \& Koutroubas, S.D. 2002. Dry matter and N accumulation and translocation for Indica and Japonica rice under Mediterranean conditions. Field Crops Research 74: 93-101.

Sarkar, M.A.; Murayama, S.; Akamine, H. \& Nakamura, I. 2002. Effect of nitrogen fertilization on photosynthetic characters and dry matter production in F-1 hybrids of rice (Oryza sativa L.). Plant Production Science 5: 131-138.

Silveira, J.A.G. \& Machado, E.C. 1990. Mobilização de nitrogênio e de carboidratos durante o desenvolvimento de panículas de duas cultivares de arroz. Revista Brasileira de Fisiologia Vegetal 2: 37-46.

Sinclair, T.R. \& Horie, T. 1989. Leaf nitrogen, photosynthesis, and crop radiation use efficiency: a review. Crop Science 29: 90-98.

Usberti Filho, J.A.; Azzini, L.E.; Camargo, O.A.B.; Soave, J.; Schmidt, N.C.; Villela, O.V.; Gallo, P.B.; Sakai, M.; Tisselli Filho, O.; Yanai, K. \& Vitti, P. 1986. IAC 4440: Novo cultivar de arroz irrigado para o estado de São Paulo. Campinas, IAC. (Boletim Técnico, 107).

Vieira, A.R.R.; Steinmetz, S. \& Brunini, O. 1990. Parâmetros de crescimento de duas cultivares de arroz sob diferentes regimes de água no solo. Pesquisa Agropecuária Brasileira 6: 897-904.

Villar, R.; Marañón, T.; Quero, J.L.; Panadero, P.; Arenas, F. \& Lambers, H. 2005. Variation in relative growth rate of 20 Aegilops (Poaceae) in the field: The importance of net assimilation rate or specific leaf depends on the time scale. Plant and Soil 272: 11-27. 